\title{
Kruppa Equation Revisited: Its Renormalization and Degeneracy
}

\author{
Yi Ma`, René Vidal, Jana Košecká, and Shankar Sastry \\ Department of Electrical Engineering and Computer Sciences \\ University of California at Berkeley, Berkeley, CA 94720-1774 \\ \{mayi, rvidal, janka, sastry\}@robotics.eecs.berkeley.edu
}

\begin{abstract}
In this paper, we study general questions about the solvability of the Kruppa equations and show that, in several special cases, the Kruppa equations can be renormalized and become linear. In particular, for cases when the camera motion is such that its rotation axis is parallel or perpendicular to translation, we can obtain linear algorithms for self-calibration. A further study of these cases not only reveals generic difficulties with degeneracy in conventional self-calibration methods based on the nonlinear Kruppa equations, but also clarifies some incomplete discussion in the literature about the solutions of the Kruppa equations. We demonstrate that Kruppa equations do not provide sufficient constraints on camera calibration and give a complete account of exactly what is missing in Kruppa equations. In particular, a clear relationship between the Kruppa equations and chirality is revealed. The results then resolve the discrepancy between the Kruppa equations and the necessary and sufficient condition for a unique calibration. Simulation results are presented for evaluation of the sensitivity and robustness of the proposed linear algorithms.
\end{abstract}

Keywords: Camera self-calibration, Kruppa equations, renormalization, degeneracy, chirality.

\section{Introduction}

The problem of camera self-calibration refers to the problem of obtaining intrinsic parameters of a camera using only information from image measurements, without any a priori knowledge about the motion between frames and the structure of the observed scene. The original question of determining whether the image measurements only are sufficient for obtaining intrinsic parameters of a camera was initially answered in [11. The proposed approach and solution utilize invariant properties of the image of the so called absolute conic. Since the absolute conic is invariant under Euclidean transformations (i.e., its representation is independent of the position of the camera) and depends only on the camera intrinsic parameters, the recovery of the image of the absolute conic is

\footnotetext{
* This work is supported by ARO under the MURI grant DAAH04-96-1-0341.
} 
then equivalent to the recovery of the camera intrinsic parameter matrix. The constraints on the absolute conic are captured by the so called Kruppa equations initially discovered by Kruppa in 1913. In Section 3 , we will provide a much more concise derivation of the Kruppa equations.

Certain algebraic and numerical approaches for solving the Kruppa equations were first discussed in [11. Some alternative and additional schemes have been explored in 717 . Nevertheless, it has been well-known that, in the presence of noise, these Kruppa equation based approaches are not guaranteed to provide a good estimate of the camera calibration and many erroneous solutions will occur [1]. Because of this, we decide to revisit the Kruppa equation based approach in this paper. More specifically, we address the following two questions:

1. Under what conditions do the Kruppa equations become degenerate or illconditioned?

2. When conditions for degeneracy are satisfied, how do the self-calibration algorithms need to be modified?

In this paper, we show that the answer to the former question is rather unfortunate: for camera motions such that the rotation axis is parallel or perpendicular to the translation, the Kruppa equations become degenerate. This explains why conventional approaches to self-calibration based on the (nonlinear) Kruppa equations often fail. Most practical images are, in fact, taken through motions close to these two types. The parallel case shows up very frequently in motion of aerial mobile robots such as an helicopter. The perpendicular case is interesting in robot navigation, where the main rotation of the on-board camera is yaw and pitch, whose axes are perpendicular to the direction of robot heading. Nevertheless, in this paper, we take one step further to show that when such motions occur, the corresponding Kruppa equations can be renormalized and become linear! This fact allows us to correct (or salvage) classical Kruppa equation based self-calibration algorithms so as to obtain much more stable linear self-calibration algorithms, other than the pure rotation case known to Hartley [4]. Our study also clarifies and completes previous analysis and results in the literature regarding the solutions of the Kruppa equations [17. This is discussed in Section 3.2 .

Relations to Previous Works: Besides the Kruppa equation based self calibration approach, alternative methods have also been studied extensively. For example some of them use the so called absolute quadric constraints [16], modulus constraints [13] and chirality constraints [5]. Some others restrict to special cases such as stationary camera [4] or to time-varying focallength 6 14. We hope that, by a more detailed study of the Kruppa equations, we may gain a better understanding of the relationships among the various selfcalibration methods. This is discussed in Section 3.3 .

\section{Epipolar Geometry Basics}

To introduce the notation, we first review in this section the well-known epipolar geometry and some properties of fundamental matrix to aid the derivation and study of Kruppa equations. 
The camera motion is represented by $(R, p)$ where $R$ is a rotation matrix as an element in the special orthogonal group $S O(3)$ and $p \in \mathbb{R}^{3}$ is a three dimensional vector representing the translation of the camera. That is, $(R, p)$ represents a rigid body motion as an element in the special Euclidean group $S E(3)$. The three dimensional coordinates (with respect to the camera frame) of a generic point $q$ in the world are related by the following Euclidean transformation:

$$
q\left(t_{2}\right)=R\left(t_{2}, t_{1}\right) q\left(t_{1}\right)+p\left(t_{2}, t_{1}\right), \quad \forall t_{1}, t_{2} \in \mathbb{R}
$$

We use the matrix $A \in \mathbb{R}^{3 \times 3}$ to represent the intrinsic parameters of the camera, which we also refer to as the calibration matrix of the camera. In this paper, without loss of generality, we will assume $\operatorname{det}(A)=1$, i.e., $A$ is an element in the special linear group $S L(3) . S L(3)$ is the group consisting of $3 \times 3$ real matrices with determinant equal to 1 . This choice of $A$ is slightly different from (and more general than) the traditional choice in the literature, but, mathematically, it is more natural to deal with. Then the (uncalibrated) image $\mathbf{x}$ (on the image plane in $\mathbb{R}^{3}$ ) of the point $q$ at time $t$ is given through the following equation:

$$
\lambda(t) \mathbf{x}(t)=A q(t), \quad \forall t \in \mathbb{R}
$$

where $\lambda(t) \in \mathbb{R}$ is a scalar encoding the depth of the point $q$. Note that this model does not differentiate the spherical or perspective projection.

Since we primarily consider the two-view case in this paper, to simplify the notation, we will drop the time dependency from the motion $\left(R\left(t_{2}, t_{1}\right), p\left(t_{2}, t_{1}\right)\right)$ and simply denote it as $(R, p)$, and also use $\mathbf{x}_{1}, \mathbf{x}_{2}$ as shorthand for $\mathbf{x}\left(t_{1}\right), \mathbf{x}\left(t_{2}\right)$ respectively. Also, for a three dimensional vector $p \in \mathbb{R}^{3}$, we can always associate to it a skew symmetric matrix $\widehat{p} \in \mathbb{R}^{3 \times 3}$ such that $p \times q=\widehat{p} q$ for all $q \in \mathbb{R}^{3} 1$

Then it is well known that the two image points $\mathbf{x}_{1}$ and $\mathbf{x}_{2}$ must satisfy the so called epipolar constraint:

$$
\mathbf{x}_{1}^{T} A^{-T} R^{T} \widehat{p} A^{-1} \mathbf{x}_{2}=0 .
$$

The matrix $F=A^{-T} R^{T} \widehat{p} A^{-1} \in \mathbb{R}^{3 \times 3}$ is the so called fundamental matrix in Computer Vision literature. When $A=I$, the fundamental matrix simply becomes $R^{T} \widehat{p}$ which is called essential matrix and plays a very important role in motion recovery [10. The following simple but extremely useful lemma will allow us to write the fundamental matrix in a more convenient form:

Lemma 1 (Hat Operator). If $p \in \mathbb{R}^{3}$ and $A \in S L(3)$, then $A^{T} \widehat{p} A=\widehat{A^{-1} p}$.

Proof: $\quad$ Since both $A^{T} \widehat{(\cdot)} A$ and $\widehat{A^{-1}(\cdot)}$ are linear maps from $\mathbb{R}^{3}$ to $\mathbb{R}^{3 \times 3}$, using the fact that $\operatorname{det}(A)=1$, one may directly verify that these two linear maps are equal on the bases: $(1,0,0)^{T},(0,1,0)^{T}$ or $(0,0,1)^{T}$.

${ }^{1}$ In the computer vision literature, such a skew symmetric matrix is also often denoted as $p_{\times}$. But we here use the notation consistent to robotics and matrix Lie group theory, where $\widehat{p}$ is used to denote to elements in the Lie algebra $s o(3)$ of $S O(3)$. 
This simple lemma will be frequently used throughout the paper. By this lemma, we have:

$$
F=A^{-T} R^{T} \widehat{p} A^{-1}=A^{-T} R^{T} A^{T} A^{-T} \widehat{p} A^{-1}=A^{-T} R^{T} A^{T} \widehat{p^{\prime}}
$$

where $p^{\prime}=A p \in \mathbb{R}^{3}$ is the so called epipole. This equation in fact has a more fundamental interpretation: an uncalibrated camera in a calibrated world is mathematically equivalent to a calibrated camera in an uncalibrated world (for more details see [9]). As we will soon see, the last form of the fundamental matrix in the above equation is the most useful one for deriving and solving the Kruppa equations.

\section{The Kruppa Equations}

Without loss of generality, we may assume that both the rotation $R$ and translation $p$ are non-trivial, i.e., $R \neq I$ and $p \neq 0$ hence the epipolar constraint (3) is not degenerate and the fundamental matrix can be estimated. The camera self-calibration problem is then reduced to recovering the symmetric matrix $\omega=A^{-T} A^{-1}$ or $\omega^{-1}=A A^{T}$ from fundamental matrices. It can be shown, even if we have chosen $A$ to be an arbitrary element in $S L(3), A$ can only be recovered up to a rotation, i.e., as an element in the quotient space $S L(3) / S O(3)$, for more details see $[9$. Note that $S L(3) / S O(3)$ is only a 5 -dimensional space. From the fundamental matrix, the epipole vector $p^{\prime}$ can be directly computed (up to an arbitrary scale) as the null space of $F$. Given a fundamental matrix $F=A^{-T} R^{T} A^{T} \widehat{p^{\prime}}$, its scale, usually denoted as $\lambda$, is defined as the norm of $p^{\prime}$. If $\lambda=\left\|p^{\prime}\right\|=1$, such a $F$ is called a normalized fundamental matrix 2 For now, we assume that the fundamental matrix $F$ happens to be normalized.

Suppose the standard basis of $\mathbb{R}^{3}$ is $e_{1}=(1,0,0)^{T}, e_{2}=(0,1,0)^{T}, e_{3}=$ $(0,0,1)^{T} \in \mathbb{R}^{3}$. Now pick any rotation matrix $R_{0} \in S O(3)$ such that $R_{0} p^{\prime}=e_{3}$. Using Lemma 1, we have $\widehat{p^{\prime}}=R_{0}^{T} \widehat{e_{3}} R_{0}$. Define matrix $D \in \mathbb{R}^{3 \times 3}$ to be:

$$
D=F R_{0}^{T}=A^{-T} R^{T} A^{T} R_{0}^{T} \widehat{e_{3}}=A^{-T} R^{T} A^{T} R_{0}^{T}\left(e_{2},-e_{1}, 0\right) .
$$

Then $D$ has the form $D=\left(\xi_{1}, \xi_{2}, 0\right)$ with $\xi_{1}, \xi_{2} \in \mathbb{R}^{3}$ being the first and second column vectors of $D$. We then have $\xi_{1}=A^{-T} R^{T} A^{T} R_{0}^{T} e_{2}, \xi_{2}=-A^{-T} R^{T} A^{T} R_{0}^{T} e_{1}$. Define vectors $\eta_{1}, \eta_{2} \in \mathbb{R}^{3}$ as $\eta_{1}=-R_{0}^{T} e_{1}, \eta_{2}=R_{0}^{T} e_{2}$, then it is direct to check that $\omega^{-1}$ satisfies:

$$
\xi_{1}^{T} \omega^{-1} \xi_{1}=\eta_{2}^{T} \omega^{-1} \eta_{2}, \quad \xi_{2}^{T} \omega^{-1} \xi_{2}=\eta_{1}^{T} \omega^{-1} \eta_{1}, \quad \xi_{1}^{T} \omega^{-1} \xi_{2}=\eta_{1}^{T} \omega^{-1} \eta_{2} .
$$

We thus obtain three homogeneous constraints on the matrix $\omega^{-1}$, the inverse (dual) of the matrix (conic) $\omega$. These constraints can be used to compute $\omega^{-1}$ hence $\omega$.

The above derivation is based on the assumption that the fundamental matrix $F$ is normalized, i.e., $\left\|p^{\prime}\right\|=1$. However, since the epipolar constraint is homogeneous in the fundamental matrix $F$, it can only be determined up to an arbitrary

\footnotetext{
${ }^{2}$ Here $\|\cdot\|$ represents the standard 2-norm.
} 
scale. Suppose $\lambda$ is the length of the vector $p^{\prime} \in \mathbb{R}^{3}$ in $F=A^{-T} R^{T} A^{T} \widehat{p^{\prime}}$. Consequently, the vectors $\xi_{1}$ and $\xi_{2}$ are also scaled by the same $\lambda$. Then the ratio between the left and right hand side quantities in each equation of (6) is equal to $\lambda^{2}$. This gives two equations on $\omega^{-1}$, the so called Kruppa equations (after its initial discovery by Kruppa in 1913):

$$
\lambda^{2}=\frac{\xi_{1}^{T} \omega^{-1} \xi_{1}}{\eta_{2}^{T} \omega^{-1} \eta_{2}}=\frac{\xi_{2}^{T} \omega^{-1} \xi_{2}}{\eta_{1}^{T} \omega^{-1} \eta_{1}}=\frac{\xi_{1}^{T} \omega^{-1} \xi_{2}}{\eta_{1}^{T} \omega^{-1} \eta_{2}} .
$$

Alternative means of obtaining the Kruppa equations are by utilizing algebraic relationships between projective geometric quantities [11] or via SVD characterization of $F[3$. Here we obtain the same equations from a quite different approach. Equation (7) further reveals the geometric meaning of the Kruppa ratio $\lambda^{2}$ : it is the square of the length of the vector $p^{\prime}$ in the fundamental matrix $F$. This discovery turns out to be quite useful when we later discuss the renormalization of Kruppa equations. In general, each fundamental matrix provides at most two algebraic constraints on $\omega^{-1}$, if the two equations in (7) happen to be independent. Since the symmetric matrix $\omega$ has five degrees of freedom, in general at least three fundamental matrices are needed to uniquely determine $\omega$. Nevertheless, as we will soon see, this is not the case for many special camera motions.

Comment 1 One must be aware that solving Kruppa equations for camera calibration is not equivalent to the camera self-calibration problem in the sense that there may exist solutions of Kruppa equations which are not solutions of a "valid" self-calibration. Given a non-critical set of camera motions, the associated Kruppa equations do not necessarily give enough constraints to solve for the calibration matrix A. See Section 3.3 for a complete account.

The above derivation of Kruppa equations is straightforward, but the expression (7) depends on a particular choice of the rotation matrix $R_{0}$ - note that such a choice is not unique. However, there is an even simpler way to get an equivalent expression for the Kruppa equations in a matrix form. Given a normalized fundamental matrix $F=A^{-T} R^{T} A^{T} \widehat{p^{\prime}}$, it is then straightforward to check that $\omega^{-1}=A A^{T}$ must satisfy the following equation:

$$
F^{T} \omega^{-1} F=\widehat{p^{\prime}} \omega^{-1} \widehat{p^{\prime}}
$$

We call this equation the normalized matrix Kruppa equation. It is readily seen that this equation is equivalent to (6). If $F$ is not normalized and is scaled by $\lambda \in \mathbb{R}$, i.e., $F=\lambda A^{-T} R^{T} A^{T} \widehat{p^{\prime}} 3$ we then have the matrix Kruppa equation:

$$
F^{T} \omega^{-1} F=\lambda^{2}{\widehat{p^{\prime}}}^{T} \omega^{-1} \widehat{p^{\prime}}
$$

This equation is equivalent to the scalar version given by (7) and is independent of the choice of the rotation matrix $R_{0}$. In fact, the matrix form reveals that the nature of Kruppa equations is nothing but inner product invariants of the group $A S O(3) A^{-1}$ (for more details see [9]).

\footnotetext{
${ }^{3}$ Without loss of generality, from now on, we always assume $\left\|p^{\prime}\right\|=1$.
} 


\subsection{Solving Kruppa Equations}

Algebraic properties of Kruppa equations have been extensively studied (see e.g. [11 17]). However, conditions on dependency among Kruppa equations obtained from the fundamental matrix have not been fully discovered. Therefore it is hard to tell in practice whether a given set of Kruppa equations suffice to guarantee a unique solution for calibration. As we will soon see in this section, for very rich classes of camera motions which commonly occur in many practical applications, the Kruppa equations will become degenerate. Moreover, since the Kruppa equations (7) or (9) are highly nonlinear in $\omega^{-1}$, most self-calibration algorithms based on directly solving these equations suffer from being computationally expensive or having multiple local minima [17]. These reasons have motivated us to study the geometric nature of Kruppa equations in order to gain a better understanding of the difficulties commonly encountered in camera self-calibration. Our attempt to resolve these difficulties will lead to simplified algorithms for selfcalibration. These algorithms are linear and better conditioned for these special classes of camera motions.

Given a fundamental matrix $F=A^{-T} R^{T} A^{T} \widehat{p^{\prime}}$ with $p^{\prime}$ of unit length, the normalized matrix Kruppa equation (8) can be rewritten in the following way:

$$
{\widehat{p^{\prime}}}^{T}\left(\omega^{-1}-A R A^{-1} \omega^{-1} A^{-T} R^{T} A^{T}\right) \widehat{p^{\prime}}=0 .
$$

According to this form, if we define $C=A^{-T} R^{T} A^{T}$, a linear (Lyapunov) map $\sigma: \mathbb{R}^{3 \times 3} \rightarrow \mathbb{R}^{3 \times 3}$ as $\sigma: X \mapsto X-C^{T} X C$, and a linear map $\tau: \mathbb{R}^{3 \times 3} \rightarrow \mathbb{R}^{3 \times 3}$ as $\tau: Y \mapsto{\widehat{p^{\prime}}}^{T} Y \widehat{p^{\prime}}$, then the solution $\omega^{-1}$ of equation (10) is exactly the (symmetric real) kernel of the composition map:

$$
\tau \circ \sigma: \quad \mathbb{R}^{3 \times 3} \stackrel{\sigma}{\longrightarrow} \mathbb{R}^{3 \times 3} \stackrel{\tau}{\longrightarrow} \mathbb{R}^{3 \times 3} .
$$

This interpretation of Kruppa equations clearly decomposes effects of the rotational and translational parts of the motion: if there is no translation i.e., $p=0$, then there is no map $\tau$; if the translation is non-zero, the kernel is enlarged due to the composition with map $\tau$. In general, the symmetric real kernel of the composition map $\tau \circ \sigma$ is 3 dimensional - while the kernel of $\sigma$ is only 2 dimensional (see [9]). The solutions for the unnormalized Kruppa are much more complicated due to the unknown scale $\lambda$. However, we have the following lemma to simplify things a little bit.

Lemma 2. Given a fundamental matrix $F=A^{-T} R^{T} A^{T} \widehat{p^{\prime}}$ with $p^{\prime}=A p$, a real symmetric matrix $X \in \mathbb{R}^{3 \times 3}$ is a solution of $F^{T} X F=\lambda^{2}{\widehat{p^{\prime}}}^{T} X{\widehat{p^{\prime}}}^{\prime}$ if and only if $Y=A^{-1} X A^{-T}$ is a solution of $E^{T} Y E=\lambda^{2} \widehat{p}^{T} Y \hat{p}$ with $E=R^{T} \widehat{p}$.

Using Lemma1, the proof of this lemma is simply algebraic. This simple lemma, however, states a very important fact: given a set of fundamental matrices $F_{i}=$ $A^{-T} R_{i}^{T} A^{T} \widehat{p_{i}^{\prime}}$ with $p_{i}^{\prime}=A p_{i}, i=1, \ldots, n$, there is a one-to-one correspondence between the set of solutions of the equations: $F_{i}^{T} X F_{i}=\lambda_{i}^{2}{\widehat{p_{i}^{\prime}}}^{T} X{\widehat{p_{i}^{\prime}}}_{i}, i=1, \ldots, n$ 
and the set of solutions of the equations: $E_{i}^{T} Y E_{i}=\lambda_{i}^{2} \widehat{p}_{i}^{T} Y \widehat{p}_{i}, i=1, \ldots, n$ where $E_{i}=R_{i}^{T} \widehat{p}_{i}$ are essential matrices associated to the given fundamental matrices. Note that these essential matrices are determined only by the camera motion. Therefore, the conditions of uniqueness of the solution of Kruppa equations only depend on the camera motion. Our next task is then to study how the solutions of Kruppa equations depend on the camera motion.

\subsection{Renormalization and Degeneracy of Kruppa Equations}

From the derivation of the Kruppa equations (7) or (9), we observe that the reason why they are nonlinear is that we do not usually know the scale $\lambda$. It is then helpful to know under what conditions the matrix Kruppa equation will have the same solutions as the normalized one, i.e., with $\lambda$ set to 1 . Here we will study two special cases for which we are able to know directly what the missing $\lambda$ is. The fundamental matrix can then be renormalized and we can therefore solve the camera calibration from the normalized matrix Kruppa equations, which are linear! These two cases are when the rotation axis is parallel or perpendicular to the translation. That is, if the motion is represented by $(R, p) \in S E(3)$ and the unit vector $u \in \mathbb{R}^{3}$ is the axis of $R$ then the two cases are when $u$ is parallel or perpendicular to $p$. As we will soon see, these two cases are of great theoretical importance: Not only does the calibration algorithm become linear, but it also reveals certain subtleties of the Kruppa equations and explains when the nonlinear Kruppa equations are most likely to become ill-conditioned.

Lemma 3. Consider a camera motion $(R, p) \in S E(3)$ where $R=e^{\widehat{u} \theta}, \theta \in(0, \pi)$ and the axis $u \in \mathbb{R}^{3}$ is parallel or perpendicular to $p$. If $\gamma \in \mathbb{R}$ and positive definite matrix $Y$ are a solution to the matrix Kruppa equation: $\widehat{p}^{T} R Y R^{T} \widehat{p}=\gamma^{2} \widehat{p}^{T} Y \widehat{p}$ associated to the essential matrix $R^{T} \widehat{p}$, then we must have $\gamma^{2}=1$. Consequently, $Y$ is a solution of the normalized matrix Kruppa equation: $\widehat{p}^{T} R Y R^{T} \widehat{p}=\widehat{p}^{T} Y \hat{p}$.

Proof: Without loss of generality we assume $\|p\|=1$. For the parallel case, let $x \in \mathbb{R}^{3}$ be a vector of unit length in the plane spanned by the column vectors of $\widehat{p}$. All such $x$ lie on a unit circle. There exists $x_{0} \in \mathbb{R}^{3}$ on the circle such that $x_{0}^{T} Y x_{0}$ is maximum. We then have $x_{0}^{T} R Y R^{T} x_{0}=\gamma^{2} x_{0}^{T} Y x_{0}$, hence $\gamma^{2} \leq 1$. Similarly, if we pick $x_{0}$ such that $x_{0}^{T} Y x_{0}$ is minimum, we have $\gamma^{2} \geq 1$. Therefore, $\gamma^{2}=1$. For the perpendicular case, since the columns of $\widehat{p}$ span the subspace which is perpendicular to the vector $p$, the eigenvector $u$ of $R$ is in this subspace. Thus we have: $u^{T} R Y R^{T} u=\gamma^{2} u^{T} Y u \Rightarrow$ $u^{T} Y u=\gamma^{2} u^{T} Y u$. Hence $\gamma^{2}=1$ if $Y$ is positive definite.

Combining Lemma 3 and Lemma 2, we immediately have:

Theorem 1 (Renormalization of Kruppa Equations). Consider an unnormalized fundamental matrix $F=A^{-T} R^{T} A^{T} \widehat{p^{\prime}}$ where $R=e^{\widehat{u} \theta}, \theta \in(0, \pi)$ and the axis $u \in \mathbb{R}^{3}$ is parallel or perpendicular to $p=A^{-1} p^{\prime}$. Let $e=p^{\prime} /\left\|p^{\prime}\right\|$. Then if $\lambda \in \mathbb{R}$ and a positive definite matrix $\omega$ are a solution to the matrix Kruppa equation: $F^{T} \omega^{-1} F=\lambda^{2} \widehat{e}^{T} \omega^{-1} \widehat{e}$, we must have $\lambda^{2}=\left\|p^{\prime}\right\|^{2}$.

\footnotetext{
${ }^{4} R$ can always be written of the form $R=e^{\widehat{u} \theta}$ for some $\theta \in[0, \pi]$ and $u \in \mathbb{S}^{2}$.
} 
This theorem claims that, for the two types of special motions considered here, there is no solution for $\lambda$ in the Kruppa equation (9) besides the true scale of the fundamental matrix. Hence we can decompose the problem into finding $\lambda$ first and then solving for $\omega$ or $\omega^{-1}$. The following theorem allows to directly compute the scale $\lambda$ for a given fundamental matrix:

Theorem 2 (Renormalization of Fundamental Matrix). Given an unnormalized fundamental matrix $F=\lambda A^{-T} R^{T} A^{T} \widehat{p^{\prime}}$ with $\left\|p^{\prime}\right\|=1$, if $p=A^{-1} p^{\prime}$ is parallel to the axis of $R$, then $\lambda^{2}$ is $\left\|F \widehat{p}^{\prime} F^{T}\right\|$, and if $p$ is perpendicular to the axis of $R$, then $\lambda$ is one of the two non-zero eigenvalues of ${\widehat{F p^{\prime}}}^{T}$.

Proof: Note that, since $\widehat{p^{\prime} \widehat{p}^{\prime}}$ is a projection matrix to the plane spanned by the column vectors of $\widehat{p^{\prime}}$, we have the identity $\widehat{p}^{\prime} \widehat{p}^{\prime} \widehat{p}^{\prime}=\widehat{p}^{\prime}$. First we prove the parallel case. It is straightforward to check that, in general, $F \widehat{p^{\prime}} F^{T}=\lambda^{2} \widehat{A R^{T} p}$. Since the axis of $R$ is parallel to $p$, we have $R^{T} p=p$ so that $\widehat{F p^{\prime} F^{T}}=\lambda^{2} \widehat{p^{\prime}}$. For the perpendicular case, let $u \in \mathbb{R}^{3}$ be the axis of $R$. By assumption $p=A^{-1} p^{\prime}$ is perpendicular to $u$. Then there exists $v \in \mathbb{R}^{3}$ such that $u=\widehat{p} A^{-1} v$. Then it is direct to check that $\widehat{p^{\prime}} v$ is the eigenvector of $F_{p^{\prime}}{ }^{T}$ corresponding to the eigenvalue $\lambda$.

Then for these two types of special motions, the associated fundamental matrix can be immediately normalized by being divided by the scale $\lambda$. Once the fundamental matrices are normalized, the problem of finding the calibration matrix $\omega^{-1}$ from normalized matrix Kruppa equations (8) becomes a simple linear one! A normalized matrix Kruppa equation in general imposes three linearly independent constraints on the unknown calibration matrix given by (6). However, this is no longer the case for the special motions that we are considering here.

Theorem 3 (Degeneracy of Kruppa Equations). Consider a camera motion $(R, p) \in S E(3)$ where $R=e^{\widehat{u} \theta}$ has the angle $\theta \in(0, \pi)$. If the axis $u \in \mathbb{R}^{3}$ is parallel or perpendicular to $p$, then the normalized matrix Kruppa equation: $\widehat{p}^{T} R Y R^{T} \widehat{p}=\widehat{p}^{T} Y \hat{p}$ imposes only two linearly independent constraints on the symmetric matrix $Y$.

Proof: For the parallel case, by restricting $Y$ to the plane spanned by the column vectors of $\widehat{p}$, it is a symmetric matrix $\tilde{Y}$ in $\mathbb{R}^{2 \times 2}$. The rotation matrix $R \in S O(3)$ restricted to this plane is a rotation $\tilde{R} \in S O(2)$. The normalized matrix Kruppa equation is then equivalent to $\tilde{Y}-\tilde{R} \tilde{Y} \tilde{R}^{T}=0$. Since $0<\theta<\pi$, this equation imposes exactly two constraints on the three dimensional space of $2 \times 2$ real symmetric matrices. The identity $I_{2 \times 2}$ is the only solution. Hence the normalized Kruppa equation imposes exactly two linearly independent constraints on $Y$.

For the perpendicular case, since $u$ is in the plane spanned by the column vectors of $\widehat{p}$, there exist $v \in \mathbb{R}^{3}$ such that $(u, v)$ form an orthonormal basis of the plane. Then the normalized matrix Kruppa equation is equivalent to:

$$
\widehat{p}^{T} R Y R^{T} \widehat{p}=\widehat{p}^{T} Y \widehat{p} \Leftrightarrow(u, v)^{T} R Y R^{T}(u, v)=(u, v)^{T} Y(u, v) .
$$

Since $R^{T} u=u$, the above matrix equation is equivalent to two equations $v^{T} R Y u=$ $v^{T} Y u, v^{T} R Y R^{T} v=v^{T} Y v$. These are the only two constraints given by the normalized Kruppa equation. 
According to this theorem, although we can renormalize the fundamental matrix when rotation axis and translation are parallel or perpendicular, we only get two independent constraints from the resulting (normalized) Kruppa equation corresponding to a single fundamental matrix. Hence for these motions, in general, we still need three such fundamental matrices to uniquely determine the unknown calibration. On the other hand, if we do not renormalize the fundamental matrix in these cases and directly use the unnormalized Kruppa equations (7) to solve for calibration, the two nonlinear equations in (7) are in fact algebraically dependent! Therefore, one can only get one constraint, as opposed to the expected two, on the unknown calibration $\omega^{-1}$. This is summarized in Table 1

Table 1. Dependency of Kruppa equation on angle $\phi \in[0, \pi)$ between the rotation and translation.

\begin{tabular}{|c|c|c|}
\hline Cases & Type of Constraints & \# of Constraints on $\omega^{-1}$ \\
\hline \hline \multirow{2}{*}{$(\phi \neq 0)$ and $\left(\phi \neq \frac{\pi}{2}\right)$} & Unnormalized Kruppa Equation & 2 \\
\cline { 2 - 3 } & Normalized Kruppa Equation & 3 \\
\hline \multirow{2}{*}{$(\phi=0)$ or $\left(\phi=\frac{\pi}{2}\right)$} & Unnormalized Kruppa Equation & 1 \\
\cline { 2 - 3 } & Normalized Kruppa Equation & 2 \\
\hline
\end{tabular}

Although, mathematically, motion involving translation either parallel or perpendicular to the rotation is only a zero-measure subset of $S E(3)$, they are very commonly encountered in applications: Many images sequences are usually taken by moving the camera around an object in trajectory composed of planar motion or orbital motion, in which case the rotation axis and translation direction are likely perpendicular to each other. Another example is a so called screw motion, whose rotation axis and translation are parallel. Such a motion shows up frequently in aerial mobile motion. Our analysis shows that, for these types of motions, even if the sufficient conditions for a unique calibration are satisfied, a self-calibration algorithm based on directly solving the Kruppa equations (7) is likely to be ill-conditioned [1]. To intuitively demonstrate the practical significance of our results, we give an example in Figure 1, Our analysis reveals that in these cases, it is crucial to renormalize the Kruppa equation using Theorem 3: once the fundamental matrix or Kruppa equations are renormalized, not only is one more constraint recovered, but we also obtain linear (normalized Kruppa) equations.

Comment 2 (Solutions of the Normalized Kruppa Equations) Claims of Theorem 3 run contrary to the claims of Propositions B.5 hence B.9 in [17]: In Proposition B.5 of [17], it is claimed that the solution space of the normalized Kruppa equations when the translation is parallel or perpendicular to the rotation axis is two or three dimensional. In Theorem 3 we claim that the solution space is always four dimensional. Theorem 9 does not cover the case when the rotation angle $\theta$ is $\pi$. However, if one allows the rotation to be $\pi$, the solutions of normalized Kruppa equations are even more complicated. For example, we know $e^{\widehat{u} \pi} \widehat{p}=-\widehat{p}$ if $u$ is of unit length and parallel to $p$ (see [8]]). Therefore, if $R=e^{\widehat{u} \pi}$, the corresponding normalized Kruppa 


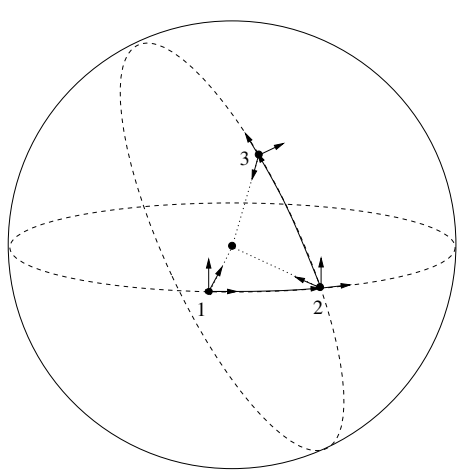

Fig. 1. Two consecutive orbital motions with independent rotations: even if pairwise fundamental matrices among the three views are considered, one only gets at most $1+1+2=4$ effective constraints on the camera intrinsic matrix if the three matrix Kruppa equations are not renormalized. After renormalization, however, we may get back to $2+2+2 \geq 5$ constraints.

equation is completely degenerate and imposes no constraints at all on the calibration matrix.

Comment 3 (Number of Solutions) Although Theorem 2 claims that for the perpendicular case $\lambda$ is one of the two non-zero eigenvalues of $\mathrm{F}_{p^{\prime}}^{T}$, unfortunately, there is no way to tell which one is the right one - simulations show that it could be either the larger or smaller one. Therefore, in a numerical algorithm, for given $n \geq 3$ fundamental matrices, one needs to consider all possible $2^{n}$ combinations. According to Theorem 1] in the noise-free case, only one of the solutions can be positive definite, which corresponds to the the true calibration.

\subsection{Kruppa Equations and Chirality}

It can be shown that if the scene is rich enough (with to come), then the necessary and sufficient condition for a unique camera calibration (see 9]) says that two general motions with rotation around different axes already determine a unique Euclidean solution for camera motion, calibration and scene structure. However, the two Kruppa equations obtained from these two motions will only give us at most four constraints on $\omega$, which is not enough to determine $\omega$ which has five degrees of freedom. We hence need to know what information is missing from the Kruppa equation. State alternatively, can we get extra independent constraints on $\omega$ from the fundamental matrix other than the Kruppa equation?

The proof of Theorem 2 suggests another equation can be derived from the fundamental matrix $F=\lambda A^{-T} R^{T} A^{T} \widehat{p^{\prime}}$ with $\left\|p^{\prime}\right\|=1$. Since $F \widehat{p^{\prime}} F^{T}=\lambda^{2} \widehat{A R^{T} p}$, we can obtain the vector $\alpha=\lambda^{2} A R^{T} p=\lambda^{2} A R^{T} A^{-1} p^{\prime}$. Then it is obvious that the following equation for $\omega=A^{-T} A^{-1}$ holds:

$$
\alpha^{T} \omega \alpha=\lambda^{4} p^{\prime T} \omega p^{\prime}
$$


Notice that this is a constraint on $\omega$, not like the Kruppa equations which are constraints on $\omega^{-1}$. Combining the Kruppa equations given in (7) with (13) we have:

$$
\lambda^{2}=\frac{\xi_{1}^{T} \omega^{-1} \xi_{1}}{\eta_{2}^{T} \omega^{-1} \eta_{2}}=\frac{\xi_{2}^{T} \omega^{-1} \xi_{2}}{\eta_{1}^{T} \omega^{-1} \eta_{1}}=\frac{\xi_{1}^{T} \omega^{-1} \xi_{2}}{\eta_{1}^{T} \omega^{-1} \eta_{2}}=\sqrt{\frac{\alpha^{T} \omega \alpha}{p^{\prime} \omega p^{\prime}}} .
$$

Is the last equation algebraically independent of the two Kruppa equations? Although it seems to be quite different from the Kruppa equations, it is in fact dependent on them. This can be shown either numerically or using simple algebraic tools such as Maple. Thus, it appears that our effort to look for extra independent, explicit constraints on $A$ from the fundamental matrix has failed 5 In the following, we will give an explanation to this by showing that not all $\omega$ which satisfy the Kruppa equations may give valid Euclidean reconstructions of both the camera motion and scene structure. The extra constraints which are missing in Kruppa equations are in fact captured by the so called chirality constraint, which was previously studied in [5]. We now give a clear and concise description between the relationship of the Kruppa equations and chirality.

Theorem 4 (Kruppa Equations and Chirality). Consider a camera with calibration matrix $I$ and motion $(R, p)$. If $p \neq 0$, among all the solutions $Y=$ $A^{-1} A^{-T}$ of the Kruppa equation $E^{T} Y E=\lambda^{2} \widehat{p}^{T} Y \hat{p}$ associated to $E=R^{T} \widehat{p}$, only those which guarantee $A R A^{-1} \in S O(3)$ may provide a valid Euclidean reconstruction of both camera motion and scene structure in the sense that any other solution pushes some plane $N \subset \mathbb{R}^{3}$ to the plane at infinity, and feature points on different sides of the plane $N$ have different signs of recovered depth.

Proof: The images $\mathbf{x}_{2}, \mathbf{x}_{1}$ of any point $q \in \mathbb{R}^{3}$ satisfy the coordinate transformation:

$$
\lambda_{2} \mathbf{x}_{2}=\lambda_{1} R \mathbf{x}_{1}+p .
$$

If there exists $Y=A^{-1} A^{-T}$ such that $E^{T} Y E=\lambda^{2} \widehat{p}^{T} Y \widehat{p}$ for some $\lambda \in \mathbb{R}$, then the matrix $F=A^{-T} E A^{-1}=A^{-T} R^{T} A^{T} \widehat{p^{\prime}}$ is also an essential matrix with $p^{\prime}=A p$, that is, there exists $\tilde{R} \in S O(3)$ such that $F=\tilde{R}^{T} \widehat{p^{\prime}}$ (see [10] for an account of properties of essential matrices). Under the new calibration $A$, the coordinate transformation is in fact:

$$
\lambda_{2} A \mathbf{x}_{2}=\lambda_{1} A R A^{-1}\left(A \mathbf{x}_{1}\right)+p^{\prime} .
$$

Since $F=\tilde{R}^{T} \widehat{p^{\prime}}=A^{-T} R^{T} A^{T} \widehat{p}^{\prime}$, we have $A R A^{-1}=\tilde{R}+p^{\prime} v^{T}$ for some $v \in \mathbb{R}^{3}$. Then the above equation becomes: $\lambda_{2} A \mathbf{x}_{2}=\lambda_{1} \tilde{R}\left(A \mathbf{x}_{1}\right)+\lambda_{1} p^{\prime} v^{T}\left(A \mathbf{x}_{1}\right)+p^{\prime}$. Let $\beta=$ $\lambda_{1} v^{T}\left(A \mathbf{x}_{1}\right) \in \mathbb{R}$, we can further rewrite the equation as:

$$
\lambda_{2} A \mathbf{x}_{2}=\lambda_{1} \tilde{R} A \mathbf{x}_{1}+(\beta+1) p^{\prime} .
$$

Nevertheless, with respect to the solution $A$, the reconstructed images $A \mathbf{x}_{1}, A \mathbf{x}_{2}$ and $\left(\tilde{R}, p^{\prime}\right)$ must also satisfy:

$$
\gamma_{2} A \mathbf{x}_{2}=\gamma_{1} \tilde{R} A \mathbf{x}_{1}+p^{\prime}
$$

${ }^{5}$ Nevertheless, extra implicit constraints on $A$ may still be obtained from other algebraic facts. For example, the so called modulus constraints give three implicit constraints on $A$ by introducing three extra unknowns, for more details see 13 . 
for some scale factors $\gamma_{1}, \gamma_{2} \in \mathbb{R}$. Now we prove by contradiction that $v \neq 0$ is impossible for a valid Euclidean reconstruction. Suppose that $v \neq 0$ and we define the plane $N=\left\{q \in \mathbb{R}^{3} \mid v^{T} q=-1\right\}$. Then for any $q=\lambda_{1} A \mathbf{x}_{1} \in N$, we have $\beta=-1$. Hence, from (15), $A \mathbf{x}_{1}, A \mathbf{x}_{2}$ satisfy $\lambda_{2} A \mathbf{x}_{2}=\lambda_{1} \tilde{R} A \mathbf{x}_{1}$. Since $A \mathbf{x}_{1}, A \mathbf{x}_{2}$ also satisfy (16) and $p^{\prime} \neq 0$, both $\gamma_{1}$ and $\gamma_{2}$ in (16) must be $\infty$. That is, the plane $N$ is "pushed" to the plane at infinity by the solution $A$. For points not on the plane $N$, we have $\beta+1 \neq 0$. Comparing the two equations (15) and (16), we get $\gamma_{i}=\lambda_{i} /(\beta+1), i=1,2$. Then for a point in the far side of the plane $N$, i.e., $\beta+1<0$, the recovered depth scale $\gamma$ is negative; for a point in the near side of $N$, i.e., $\beta+1>0$, the recovered depth scale $\gamma$ is positive. Thus, we must have that $v=0$.

Comment 4 (Quasi-affine Reconstruction) Theorem 4 essentially implies the chirality constraints studied in [5]. According to the above theorem, if only finitely many feature points are measured, a solution of the calibration matrix A which may allow a valid Euclidean reconstruction should induce a plane $N$ not cutting through the convex hull spanned by all the feature points and camera centers. Such a reconstruction is referred as quasi-affine in [5].

It is known that, in general, all $A$ 's which make $A R A^{-1}$ a rotation matrix form a one parameter family [9]. Thus, following Theorem4, a camera calibration can be uniquely determined by two independent rotations regardless of translation if enough feature points are available. An intuitive example is provided in Figure 2.

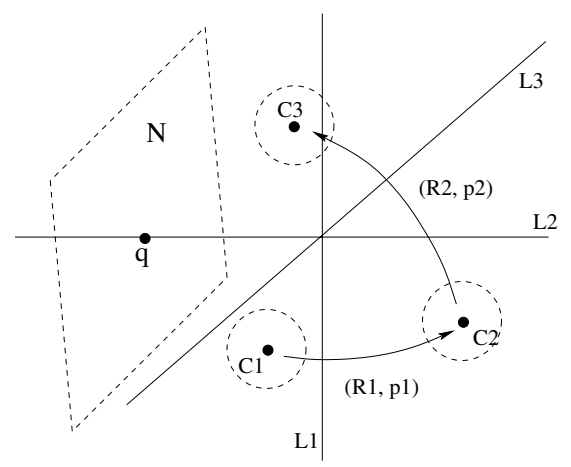

Fig. 2. A camera undergoes two motions $\left(R_{1}, p_{1}\right)$ and $\left(R_{2}, p_{2}\right)$ observing a rig consisting of three straight lines $L_{1}, L_{2}, L_{3}$. Then the camera calibration is uniquely determined as long as $R_{1}$ and $R_{2}$ have independent rotation axes and rotation angles in $(0, \pi)$, regardless of $p_{1}, p_{2}$. This is because, for any invalid solution $A$, the associated plane $N$ (see the proof of Theorem 4) must intersect the three lines at some point, say $q$. Then the reconstructed depth of point $q$ with respect to the solution $A$ would be infinite (points beyond the plane $N$ would have negative recovered depth). This gives us a criteria to exclude all such invalid solutions.

The significance of Theorem 4 is that it explains why we get only two constraints from one fundamental matrix even in the two special cases when the 
Kruppa equations can be renormalized - extra ones are imposed by the structure, not the motion. The theorem also resolves the discrepancy between the Kruppa equations and the necessary and sufficient condition for a unique calibration: the Kruppa equations, although convenient to use, do not provide sufficient conditions for a valid calibration which allows a valid Euclidean reconstruction of both the camera motion and scene structure. However, the fact given in Theorem 4 is somewhat difficult to harness in algorithms. For example, in order to exclude invalid solutions, one needs feature points on or beyond the plane $N 6$ Alternatively, if such feature points are not available, one may first obtain a projective reconstruction and then use the so called absolute quadric constraints to calibrate the camera [16]. However, in such a method, the camera motion needs to satisfy a stronger condition than requiring only two independent rotations, i.e., it cannot be critical in the sense specified in [15].

\section{Simulation Results}

In this section, we test the performance of the proposed algorithms through different experiments. The error measure between the actual calibration matrix $A$ and the estimated calibration matrix $\tilde{A}$ was chosen to be error $=\frac{\|A-\tilde{A}\|}{\|A\|} \times 100$. For all the simulations, field of view is chosen to be 90 degrees for a $500 \times 500$ pixel image size; a cloud of 20 points are randomly chosen with depths vary from 100 to 400 units of focal length; the number of trials is always 100 and the number of image frames is 3 to 4 (depending on the minimum number of frames needed by each algorithm). The calibration matrix $A$ is simply the transformation from the original $2 \times 2$ (in unit of focal length) image to the $500 \times 500$ pixel image. For these parameters, the true $A$ should be $A=\left(\begin{array}{ccc}250 & 0 & 250 \\ 0 & 250 & 250 \\ 0 & 0 & 1\end{array}\right)$. The ratio of the magnitude of translation and rotation, or simply the $T / R$ ratio, is compared at the center of the random cloud (scattered in the truncated pyramid specified by the given field of view and depth variation). For all simulations, the number of trials is 100 .

Pure rotation case: For comparison, we here also implement the linear algorithm proposed by Hartley [4 for calibrating a pure rotating camera. Figures 3 and 4 show the experiments performed in the pure rotation case. The axes of rotation are $X$ and $Y$ for Figures 3 and 4 . The amount of rotation is $20^{\circ}$. The perfect data was corrupted with zero-mean Gaussian noise with standard deviation $\sigma$ varying from 0 to 5 pixels. In Figures 3 it can be observed that the algorithm performs very well in the presence of noise, reaching errors of less than $6 \%$ for a noise level of 5 pixels. Figure 4 shows the effect of the amount of translation. This experiment is aimed to test the robustness of the pure rotation algorithm with respect to translation. The $T / R$ ratio was varied from 0 to 0.5

${ }^{6}$ Some possible ways of harnessing the constraints provided by chirality have been discussed in [5]. Basically they give inequality constraints on the possible solutions of the calibration. 
and the noise level was set to 2 pixels. It can be observed that the algorithm is not robust with respect to the amount of translation.

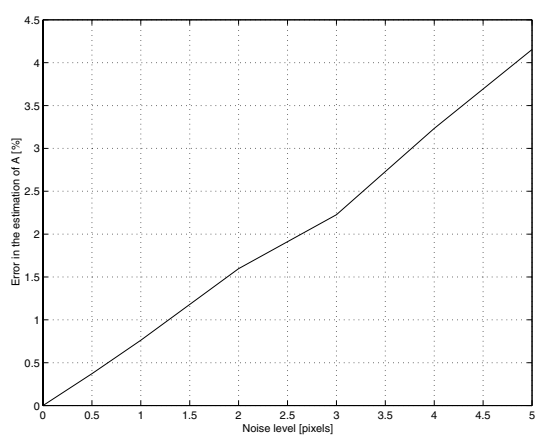

Fig. 3. Pure rotation algorithm. Rotation axes $X-Y$.

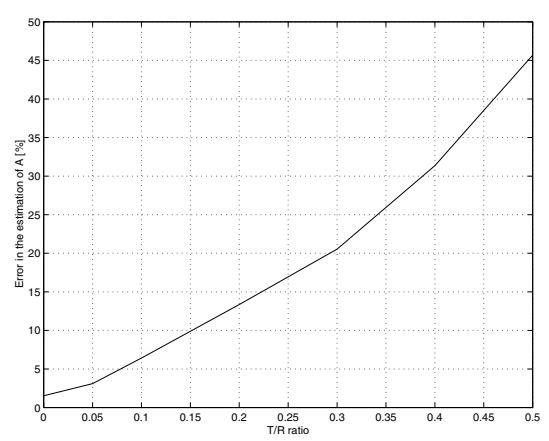

Fig. 4. Pure rotation algorithm in presence of translation. Rotation axes $X$ $Y, \sigma=2$.

Translation parallel to rotation axis: Figures [5] and 6] show the experiments performed for our algorithm 7 when translation is parallel to the axis of rotation 8 The non-isotropic normalization procedure proposed by Hartley [2] and statistically justified by Mühlich and Mester [12] was used to estimate the fundamental matrix. Figure 5 shows the effect of noise in the estimation of the calibration matrix for $T / R=1$ and a rotation of $\theta=20^{\circ}$ between consecutive frames. It can be seen that the normalization procedure improves the estimation of the calibration matrix, but the improvement is not significant. This result is consistent with that of [12, since the effect of normalization is more important for large noise levels. On the other hand, the performance of the algorithm is not as good as that of the pure rotation case, but still an error of $5 \%$ is reached for a noise level of 2 pixels. Figure 6] shows the effect of the angle of rotation in the estimation of the calibration matrix for a noise level of 2 pixels. It can be concluded that a minimum angle of rotation between consecutive frames is required for the algorithm to succeed.

Translation perpendicular to rotation axis: Figures 7 and 8 show the experiments performed for our algorithm when translation is perpendicular to the axis of rotation. It can be observed that this algorithm is much more sensitive to noise. The noise has to be less than 0.5 pixels in order to get an error of $5 \%$. Experimentally it was found that Kruppa equations are very sensitive to the

\footnotetext{
7 Although in this paper we do not outline the algorithm, it should be clear from Section 3.2

${ }^{8}$ For specifying the Rotation/Translation axes, we simply use symbols such as " $X Y$ $Y Y-Z Z$ " which means: for the first pair of images the relative motion is rotation along $X$ and translation along $Y$; for the second pair both rotation and translation are along $Y$; and for the third pair both rotation and translation are along $Z$.
} 


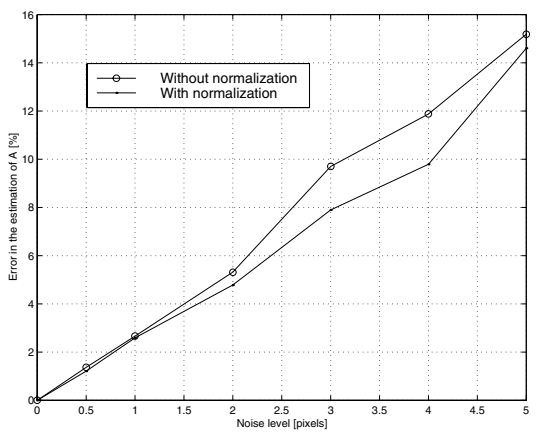

Fig. 5. Rotation parallel to translation case. $\theta=20^{\circ}$. Rotation/Translation axes: $X X-Y Y-Z Z, T / R$ ratio $=1$.

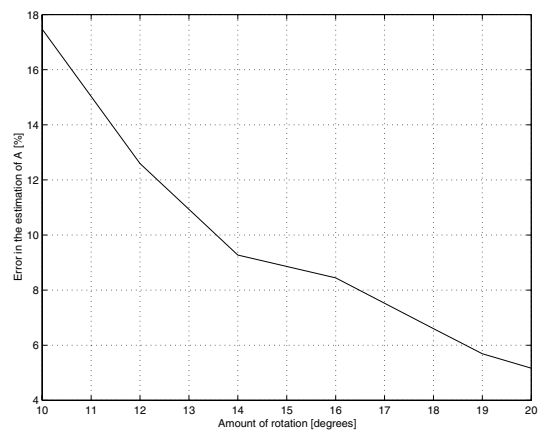

Fig. 6. Rotation parallel to translation case. $\sigma=2$. Rotation/Translation axes: $X X-Y Y-Z Z, T / R$ ratio $=1$.

normalization of the fundamental matrix $F$ and that the eigenvalues $\lambda_{1}$ and $\lambda_{2}$ of ${\widehat{F p^{\prime}}}^{T}$ are close to each other. Therefore in the presence of noise, the estimation of those eigenvalues might be ill conditioned (even complex eigenvalues are obtained) and so might the solution of Kruppa equations. Another experimental problem is that more than one non-degenerate solution to Kruppa equations can be found. This is because, when taking all possible combinations of eigenvalues of $F{\widehat{p^{\prime}}}^{T}$ in order to normalize $F$, the smallest eigenvalue of the linear map associated to "incorrect" Kruppa equations can be very small. Besides, the eigenvector associated to this eigenvalue can eventually give a non-degenerate matrix. Thus in the presence of noise, you can not distinguish between the correct and one of these incorrect solutions. The results presented here correspond to the best match to the ground truth when more than one solution is found. Finally it is important to note that large motions can significantly improve the performance of the algorithm. Figure 8 shows the error in the estimation of the calibration matrix for a rotation of $30^{\circ}$. It can be observed that the results are comparable to that of the parallel case with a rotation of $20^{\circ}$.

Robustness: We denote the angle between the rotation axis and translation by $\phi$. The two linear algorithms we have studied in the above are only supposed to work for the cases $\phi=0^{\circ}$ and $\phi=90^{\circ}$. In order to check how robust these algorithms are, we run them anyway for cases when $\phi$ varies from $0^{\circ}$ to $90^{\circ}$. The noise level is 2 pixels, amount of rotation is always $20^{\circ}$ and the $T / R$ ratio is 1. Translation and rotation axes are given by Figure 9. Surprisingly, as we can see from the results given in Figure 10, for the range $0^{\circ} \leq \phi \leq 50^{\circ}$, both algorithms give pretty close estimates. Heuristically, this is because, for this range of angle, the eigenvalues of the matrix $F{\widehat{p^{\prime}}}^{T}$ are complex and numerically their norm is very close to the norm of the matrix $F \widehat{p^{\prime}} F^{T}$. Therefore, the computed renormalization scale $\lambda$ from both algorithms is very close, as is the calibration estimate. For $\phi>50^{\circ}$, the eigenvalues of $\widehat{F \bar{p}^{\prime}}$ become real and the performance of the two algorithms is no longer the same. Near the conditions under which 


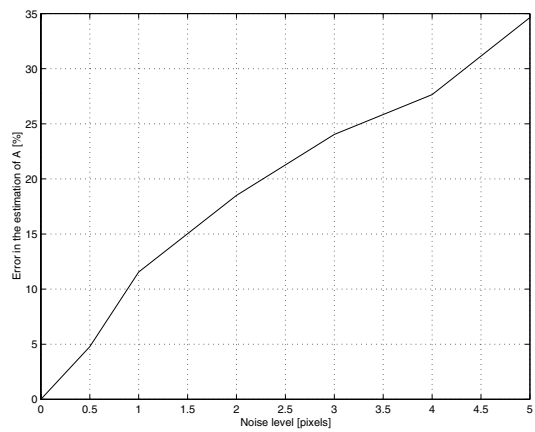

Fig. 7. Rotation orthogonal to translation case. $\theta=20^{\circ}$. Rotation/Translation axes: $X Y-Y Z-Z X$, $T / R$ ratio $=1$.

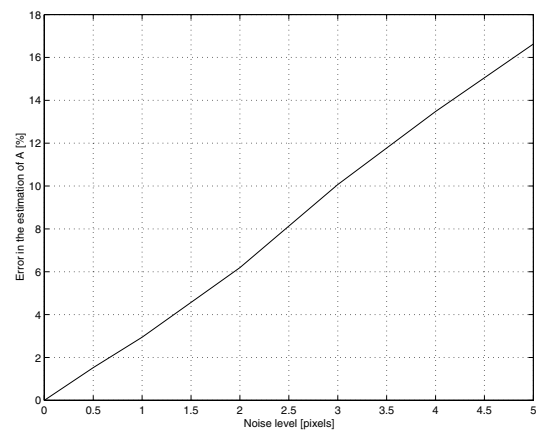

Fig. 8. Rotation orthogonal to translation case. $\theta=30^{\circ}$. Rotation/Translation axes: $X Y-Y Z-Z X$, $T / R$ ratio $=1$.

these algorithms are designed to work, the algorithm for the perpendicular case is apparently more sensitive to the perturbation in the angle $\phi$ than the one for the parallel case: As clear from the figure, a variation of $10^{\circ}$ degree of $\phi$ results an increase of error almost $50 \%$. We are currently conducting experiments on real images and trying to find ways to overcome this difficulty.

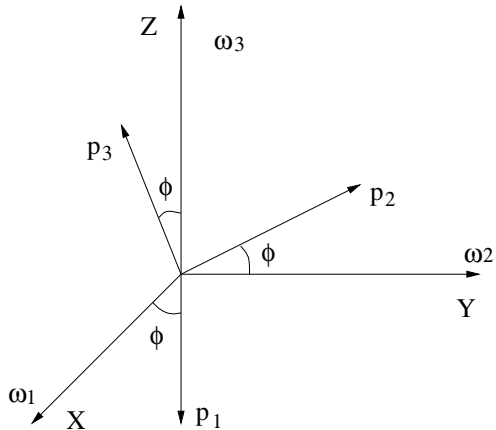

Fig. 9. The relation of the three rotation axes $\omega_{1}, \omega_{2}, \omega_{3}$ and three translations $p_{1}, p_{2}, p_{3}$.

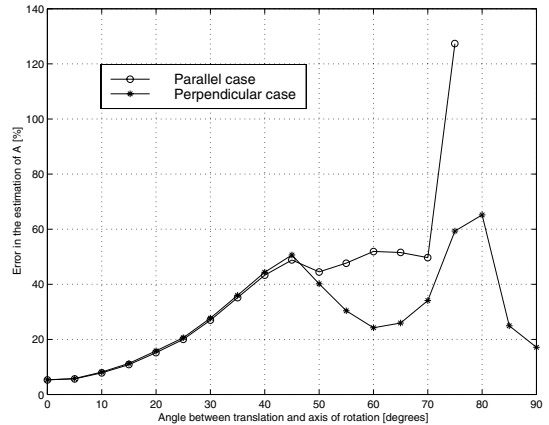

Fig. 10. Estimation error in calibration w.r.t. different angle $\phi$.

\section{Conclusions}

In this paper, we have revisited the Kruppa equations based approach for camera self-calibration. Through a detailed study of the cases when the camera rotation axis is parallel or perpendicular to the translation, we have discovered generic difficulties in the conventional self-calibration schemes based on directly solving the nonlinear Kruppa equations. Our results not only complete existing results in 
the literature regarding the solutions of Kruppa equations but also provide brand new linear algorithms for self-calibration other than the well-known one for a pure rotating camera. Simulation results show that, under the given conditions, these linear algorithms provide good estimates of the camera calibration despite the degeneracy of the Kruppa equations. The performance is close to that of the pure rotation case.

\section{References}

1. S. Bougnoux. From projective to Euclidean space under any practical situation, a criticism of self-calibration. In Proceedings of IEEE conference on Computer Vision and Pattern Recognition, pages 790-796, 1998.

2. R. I. Hartley. In defense of the 8-point algorithm. IEEE Transactions on Pattern Analysis and Machine Intelligence, 19(6), 1997.

3. R. I. Hartley. Kruppa's equations derived from the fundamental matrix. IEEE Transactions on Pattern Analysis and Machine Intelligence, 19(2):133-135, February 1997.

4. R. I. Hartley. Self-calibration of stationary cameras. International Journal of Computer Vision, 22(1):5-23, 1997.

5. R. I. Hartley. Chirality. International Journal of Computer Vision, 26(1):41-61, 1998.

6. A. Heyden and K. Astrom. Euclidean reconstruction from image sequences with varying and unknown focal length and principal point. In Proceedings of IEEE Conference on Computer Vision and Pattern Recognition, 1997.

7. Q.-T. Luong and O. Faugeras. Self-calibration of a moving camera from point correspondences and fundamental matrices. IJCV, 22(3):261-89, 1997.

8. Y. Ma, J. Košecká, and S. Sastry. Linear differential algorithm for motion recovery: A geometric approach. International Journal of Computer Vision, 36(1):71-89, 1998.

9. Y. Ma, René Vidal, J. Košecká, and S. Sastry. Camera self-calibration: geometry and algorithms. UC Berkeley Memorandum No. UCB/ERL M99/32, 1999.

10. S. J. Maybank. Theory of Reconstruction from Image Motion. Springer Series in Information Sciences. Springer-Verlag, 1993.

11. S. J. Maybank and O. D. Faugeras. A theory of self-calibration of a moving camera. International Journal of Computer Vision, 8(2):123-151, 1992.

12. M. Mühlich and R. Mester. The role of total least squares in motion analysis. In Proceedings of European Conference on Computer Vision, pages 305-321, 1998.

13. M. Pollefeys and L. Van Gool. Stratified self-calibration with the modulus constraint. IEEE Transactions on Pattern Analysis and Machine Intelligence, 21(8):707-24, 1999.

14. M. Pollefeys, R. Koch, and L. Van Gool. Self-calibration and metric reconstruction in spite of varying and unknown internal camera parameters. In Proceedings of 6th International Conference on Computer Vision, pages 90-95, 1998.

15. P. Sturm. Critical motion sequences for monocular self-calibration and uncalibrated Euclidean reconstruction. In Proceedings of IEEE conference on Computer Vision and Pattern Recognition, pages 1100-1105, 1997.

16. B. Triggs. Autocalibration and the absolute quadric. In Proceedings of IEEE conference on Computer Vision and Pattern Recognition, 1997.

17. C. Zeller and O. Faugeras. Camera self-calibration from video sequences: the Kruppa equations revisited. Research Report 2793, INRIA, France, 1996. 